\title{
Tax Compliance with Uncertain Income: a Stochastic Control Model
}

\author{
Gaetano T. Spartà;, Gabriele Stabile*
}

\begin{abstract}
This paper examines the compliance behaviour of a taxpayer endowed with a stochastic income, taking into account dynamical factors as public and private investments, within a stochastic control framework. Assuming logarithmic utilities and thanks to a suitable rewrite of the problem, we provide an existence and uniqueness result for the solution of the Hamilton-Jacobi-Bellman equation associated to the control problem, and we rely on a symbolic and numerical algorithm to study its solution. Moreover, we implement a Monte Carlo simulation in order to determine an estimate of the mean and the variance of the total declared income together with a confidence interval. To illustrate how the method works, we present a computational example where we assign values to the parameters. In this case we perform a sensitivity analysis, showing how the total declared income is affected by public and private investments, probability of being discovered, fine, tax rate and income uncertainty.
\end{abstract}

Keywords: Tax evasion, Stochastic income, Stochastic control, HJB equation.

\section{Introduction}

In this paper we analyse a stochastic control model on taxpayer's expectations (or, in an alternative interpretation, forecasting the taxpayer's behaviour), taking into account the presence of public and private investments.

The theoretical literature on tax evasion offers the seminal paper of Allingham and Sandmo (1972). They treat the choice of evading as a gamble, in which the discovered evader has to pay taxes on the concealed income at a penalty rate that is higher than the tax rate. In this framework, the taxpayer chooses the declared income in order to maximize her expected (with respect to the probability of being discovered) utility. Yitzhaki (1974) considers a model where the fine is imposed on the evaded tax, rather than on the evaded income as in the Allingham and Sandmo's model. Assuming that the taxpayer has an absolute risk aversion which decreases with income, he concludes that as the tax rate increases the income evaded decreases.

*Dipartimento di Metodi e Modelli per l'Economia, il Territorio e la Finanza, Sapienza-Università di Roma, via del Castro Laurenziano 9, 00161 Roma, Italy.

Financial support granted by Sapienza-Università di Roma, Research Project 'Tax evasion, corruption and inequality: Quantitative models, empirical evidence and corrective policies' (2015 prot. C26H159TJS), is gratefully acknowledged by the authors. 
As Allingham and Sandmo noticed, tax evasion problem "is related to the analysis of optimal portfolio and insurance policies in the economics of uncertainty". The idea is that the compliance/evasion decision can be seen as analogous to the portfolio choice with two assets, one safe and one risky. The income evaded can be regarded as the investment in the risky asset, due to the risky prospect of detection and punishment. The so called portfolio approach gave rise to a large number of contributions to the literature (see Cowell 1990; Sandmo 2005 for a comprehensive survey of the literature) which extended the original model in a number of directions. However in the literature appeared papers reporting empirical evidences in contrast with the findings of the classical Allingham-Sandmo-Yitzhaki papers. First of all, experimental evidence suggests a positive relationship between evasion and the tax rate (the so called Yitzhaki puzzle). Moreover, the classical model seems unable to explain the rate of tax compliance documented in many countries. For instance experimental literature suggests that there are people who never evade, even when the probability of detection is zero. To reconcile theory with evidence researchers have recognized that tax evasion have also non-pecuniary determinants that make evasion different from a gamble. Among the possible determinants, researchers report the individual morality, stigma, ethical norms (see Gordon 1989; Bordignon 1993; Erard and Fenstein 1994; Andreoni et al. 1998 among others). Other authors argue that social interactions may also be important to explain tax behaviors, by relying on the instruments of game theory (Torgler 2003; O'Doherty 2014).

Then, it has been developed a recent body of literature that extends the portfolio approach to tax evasion from a static to a (deterministic or stochastic) continuoustime control framework. In particular, see the work of Lin and Yang (2001) (with the comment of Dzhumashev and Gahramanov 2011) on tax evasion and stochastic growth, the paper of Chen (2003) on deterministic growth with public capital, the work of Dzhumashev (2007) on stochastic growth with corruption, the paper of Dzhumashev and Gahramanov (2008) on stochastic growth with public capital, the work of Cerqueti and Coppier (2011) on stochastic growth with public capital and a game-theoretical approach for tax evasion and corruption (see also Cerqueti and Coppier 2014), the paper of Levaggi and Menoncin (2013) with various possible choices for the utility function and the penalty form, the work of Célimène et al. (2016) on stochastic growth with corruption in an open economy.

In this paper we analyse the compliance behaviour of a taxpayer endowed with a stochastic income, taking into account the presence of public and private investments. In particular, we are interested in studying the taxpayer compliance in a short time interval (for example one or few years), where the parameters of the tax system are assumed to be constant. Our model differs in various aspects from the stochastic control literature on tax evasion. In fact, we model the taxpayer's income as a diffusion process, which is not derived by the enforcement mechanism (as in Lin and Yang 2001; Dzhumashev and Gahramanov 2011). In our model the audit probability and the fine are considered in the objective functional. Rather than study the implications of tax evasion on the economic growth, we are interested in providing 
a model able to analyze the compliance behaviour of a single taxpayer. Finally, we analyze a finite time horizon control problem, whereas Lin and Yang (2001), Levaggi and Menoncin (2013) study an infinite time horizon problem.

We model the taxpayer's income as a diffusion process depending on both public and private investments. Indeed, a part of the collective tax revenue is invested by the public authorities and it raises the future income of the taxpayer, who is more motivated to tax compliance (indirect effect). Furthermore, a part of the taxpayer's effective income is invested by herself, and it raises her future income (direct effect). The taxpayer's problem is to select the compliance policy that maximizes (in a finite time-interval) her expected well-being, sum of the utility of income and the 'confidence' function. The confidence function expresses the social responsibility of the taxpayer and her confidence in institutions. It depends, as well as on the declared income, on the tax rate and on the public expenditure effectiveness (see Spartà 2015 for an exhaustive introduction to this concept).

The Markovian structure of the problem allows us to use the dynamic programming approach. In case of logarithmic utilities and thanks to a suitable rewrite of the problem, we provide an existence and uniqueness result for the solution of the Hamilton-Jacobi-Bellman equation (HJB), and a verification theorem showing the relationship between the solution of the HJB equation and the value function of the control problem. The HJB equation takes the form of a partial differential equation which we are not able to explicitly solve. Thus we rely on a symbolic and numerical algorithm to study the HJB equation, that allow us also to provide a graphical representation of the value function. Moreover, we implement a Monte Carlo simulation in order to determine an estimate of the mean and the variance of the total declared income, together with a confidence interval. To illustrate how the method works, we present a computational example where we assign values to the parameters. We also present a sensitivity analysis, showing how the total declared income is affected by public and private investments, probability of being discovered, fine, tax rate and income uncertainty.

In Section 2, the general model is presented. In Section 3, a paradox on risk neutrality is formalized and solved. Section 4 deals with a class of models for which we study (by means of a transformation of variables) the analytical properties, and provides a computational example. Finally, Section 5 reviews the main conclusions of this paper and illustrates directions for further research.

\section{General model}

Let us consider a taxpayer whose income evolves in time according to the stochastic differential law

$$
d w_{t}=g\left(x_{t}, w_{t}\right) d t+h\left(w_{t}\right) d B_{t},
$$

with initial endowment $w_{0}>0 . B_{t}$ is a Brownian motion, in a probability space 
$(\Omega, \mathscr{F}, P)$, with augmented natural filtration $\left\{\mathscr{F}_{t}\right\}$ (that is, $\left\{\mathscr{F}_{t}\right\}$ is obtained adding the negligible events of $\mathscr{F}$ to the filtration generated by the Brownian motion).

Let us assume that 0 is the 'time of expectations' (or forecasts) and $t \in] 0, T]$ is the 'time of choices'. The citizen in the infinitesimal time interval $[t-d t, t]$ has income $w_{t} d t$ and she is called to pay taxes at a rate $\theta$, with $0<\theta<1$. If she declares $x_{t}<w_{t}$ (that is, $x_{t} d t$ represents the declared income in the infinitesimal interval $[t-d t, t])$, she can be recognized as evader with (perceived) probability equal to $p$. In such case, she has to pay a fine $f>1$ on the evaded tax $\theta\left(w_{t}-x_{t}\right) d t$, i.e. she has to pay $f \theta\left(w_{t}-x_{t}\right) d t$. Therefore, the citizen ends up with an effective income (in the infinitesimal interval $[t-d t, t]$ ) equal to $y_{t} d t$, with $y_{t}=w_{t}-\theta x_{t}$ in case she is not discovered, and $y_{t}=w_{t}-\theta x_{t}-f \theta\left(w_{t}-x_{t}\right)$ otherwise. Furthermore, the citizen has its own 'well-being function' $B$ of the form

$$
B(y, x, \theta, \alpha)=U(y)+C(x, \theta, \alpha),
$$

where $y \in] 0, \infty[$ is the effective income, $x \in] 0, \infty[$ is the declared income, $\alpha \in] 0,1[$ is a parameter of (perceived) effectiveness of public expenditure, the (real-valued) functions $U$ and $C$ are, respectively, the classical utility of income and the 'confidence' (a utility of contributing to the collective welfare).

We consider the problem of a taxpayer who forecasts her compliance (the process $x_{t}$ ) in order to maximize the following functional

$$
E_{0}\left(\int_{0}^{T} F\left(w_{t}, x_{t}\right) d t\right)
$$

where $E_{0}()$ is the conditional expectation with respect to the initial endowment $w_{0}$, and $F\left(w_{t}, x_{t}\right)$ is the expected well-being of the taxpayer, that is

$$
F\left(w_{t}, x_{t}\right)=(1-p) U\left(w_{t}-\theta x_{t}\right)+p U\left(w_{t}-\theta x_{t}-f \theta\left(w_{t}-x_{t}\right)\right)+C\left(x_{t}, \theta, \alpha\right) .
$$

The interpretation is that the taxpayer expects that in the time interval $[0, T]$ she will declare a (stochastic) total income

$$
\bar{X}_{T o t}=\int_{0}^{T} \bar{x}_{t} d t
$$

where $\bar{x}_{t}(t \in[0, T])$ denotes the optimal control process of the problem.

As in (Spartà, 2016), the model describes the expectations of the taxpayer on the part of income that she will declare in the future. However, in this paper we assume that $w_{t}$ follows the stochastic differential law (1), namely, the income has a trend driven by the function $g$ and an uncertainty driven by the function $h$. Now, the taxpayer's compliance decision becomes truly dynamic, because the trend function $g$ depends on $x_{t}$ (that is, the choice at time $t$ determines the trend of the income in the future). For instance (as we can see in the sections 3 and 4) the function $g$ can express the effect of investments (public and private) on the income. 
For technical reasons, namely to avoid dealing with the utility of negative amounts, we put a ceiling on the fine, assuming $0<\theta<\frac{1}{f}$. This hypothesis is consistent for example with tax systems characterized by frequent checks and fines not too high. We assume that the utility functions $U$ and $C$ have continuous second order derivatives with respect to all their arguments. We set $C(0, \theta, \alpha)$ and $C_{x}(0, \theta, \alpha)$ as the limit (eventually not finite) respectively of $C(x, \theta, \alpha)$ and $C_{x}(x, \theta, \alpha)$, for $x$ that tends to 0 from the right. We assume that $U$ is strictly increasing and strictly concave, i.e. for all $y$

$$
U^{\prime}(y)>0, U^{\prime \prime}(y)<0 .
$$

We assume similar hypothesis for the confidence $C$ as a function of the declared income, i.e. for all $x, \theta, \alpha$,

$$
C_{x}(x, \theta, \alpha) \geq 0, C_{x x}(x, \theta, \alpha) \leq 0 .
$$

Moreover, we also assume that there exists $\bar{\theta} \in[0,1[$ such that for $\theta>\bar{\theta}$ (and for all $x, \alpha)$ we have

$$
C_{x \theta}(x, \theta, \alpha) \leq 0
$$

namely, the marginal confidence of declared income is monotonically non-increasing with respect to $\theta$, at least above $\bar{\theta}$. This assumption seems reasonable because if taxes are perceived as too high, citizens lose confidence in institutions and then they are less motivated to give their fiscal contribution.

Furthermore, we assume that for all $x, \theta, \alpha$ we have

$$
C_{x \alpha}(x, \theta, \alpha) \geq 0
$$

that is, the marginal confidence of declared income is monotonically non-decreasing with respect to $\alpha$. Finally, we assume that the (real) functions $g, h$ are measurable (with respect to the real Borel $\sigma$-algebras).

We denote by $A$ the set of admissible control processes defined as follows

$$
A=\left\{\begin{array}{l}
x(t, \omega):[0, T] \times \Omega \rightarrow \mathbb{R} \text { such that }: \\
\text { 1) } x_{t}(\omega) \text { is measurable with respect to } \mathscr{F}_{t} \text { and } \mathscr{B}(\mathbb{R}), \text { for all } t . \\
\text { 2) There exists a Markov process } w \text { solution of }(1), \\
\text { pathwise unique and positive. } \\
\text { 3) } 0 \leq x_{t} \leq w_{t}(\text { in }[0, T] \times \Omega) . \\
\text { 4) } E_{0}\left(\int_{0}^{T} F\left(w_{t}, x_{t}\right) d t\right) \text { exists. }
\end{array}\right\}
$$

We remark that, since we are describing a general model, here we do not require any conditions on the functions $g$ and $h$ in order to ensure the existence and uniqueness of the solution of (1). Later on in Section 4.1, we present the analytical study of a particular class of models, and there we refine the definition of the set of admissible control processes.

The value function of the problem takes the form 


$$
V\left(w_{t}, t\right)=\sup _{x \in A} E_{t}\left(\int_{t}^{T} F\left(w_{s}, x_{s}\right) d s\right)
$$

(where $E_{t}()$ is the conditional expectation with respect to the initial value $w_{t}$ ). The HJB equation for the stochastic control problem (4) is

$$
\sup _{x(w i t h 0 \leq x \leq w)}\left[F(w, x)+V_{w}(w, t) g(x, w)+\frac{1}{2} V_{w w}(w, t)(h(w))^{2}+V_{t}(w, t)\right]=0,
$$

with the (terminal) condition at time $T$

$$
V(w, T)=0
$$

\section{Solution of the paradox on risk neutrality}

Let us consider the static and deterministic model in which, slightly weakening the hypotheses on the utility $U$ (in order to consider the linear case), we assume

$$
\left\{\begin{array}{l}
U\left(y_{1}\right)=k y_{1} \\
C\left(y_{2}, \theta, \alpha\right)=0
\end{array}\right.
$$

with $k>0$. Namely, the taxpayer has linear utility (that describes risk neutrality). Notice that, being the confidence identically zero, this case can also be seen as a particular specification of the classical models of Allingham and Sandmo (1972) and Yitzhaki (1974) (weakening the hypotheses on $U$ ).

Let $W$ be the gross income of the taxpayer. She has to decide how much income $X$ to report. The expected well-being takes the form (see (2) and (5))

$$
F(W, X)=(1-p) k(W-\theta X)+p k(W-\theta X-f \theta(W-X)) .
$$

In case $p f=1$, the expected well-being becomes

$$
F(W, X)=(1-\theta) k W,
$$

i.e. it is independent of $X$. Therefore, in this case all the choices of declared income $\bar{X} \in[0, W]$ are equivalent for the taxpayer. Namely, there is a paradoxical situation in which the taxpayer is indifferent with respect to her tax compliance.

In order to solve the paradox, we put the choice of the taxpayer in a dynamical context considering the presence of public investments. Let us analyze the (deterministic) control problem

$$
\sup _{x_{t}\left(\text { with } 0 \leq x_{t} \leq w_{t}\right)} \int_{0}^{1} F\left(w_{t}, x_{t}\right) d t
$$




$$
d w_{t}=\left[m+c \ln \left(1+\frac{x_{t}}{w_{t}}\right)\right] d t
$$

where

$$
F\left(w_{t}, x_{t}\right)=(1-p) k\left(w_{t}-\theta x_{t}\right)+p k\left(w_{t}-\theta x_{t}-f \theta\left(w_{t}-x_{t}\right)\right)=(1-\theta) k w_{t},
$$

with $c>0, w_{0}>0, m>-w_{0}$. This is a deterministic case (weakening the hypotheses on $U$ ) of the general control model that we have described in Section 2. The function $c \ln \left(1+\frac{x_{t}}{w_{t}}\right)$ in (6) represents the effect of public investments on the trend of income (to be more precise, it is an indirect effect, expressing that the taxpayer is motivated to declare her income by the (expected) positive effect of public investments). The constant $m$ expresses the other factors that drive the expected trend of the income. It is easy to see that the solution of the problem is $\bar{x}_{t}=w_{t}$, for all $t \in[0,1]$ (because this choice of the control function $x_{t}$ maximizes the state function $w_{t}$, and in this case the expected well-being $F$ is (strictly) increasing with respect to $w_{t}$ ). The economic interpretation is that now the compliance choices are not equivalent, but the taxpayer reports her income in full because she takes into account the effect of public investments.

We can also complete the analytical study of the problem, considering the value function

$$
\begin{aligned}
V\left(w_{t}, t\right) & =\int_{t}^{1}(1-p) k\left(w_{s}-\theta w_{s}\right)+p k\left(w_{s}-\theta w_{s}-f \theta\left(w_{s}-w_{s}\right)\right) d s \\
& =\int_{t}^{1} k(1-\theta) w_{s} d s
\end{aligned}
$$

Plugging the optimal compliance rate $\bar{x}_{t}=w_{t}$, for all $t \in[0,1]$ into the differential equation (6), we find that $w_{t}=w_{0}+[m+c \ln (2)] t$, whose primitive function is $w_{0} t+\frac{m+c \ln (2)}{2} t^{2}$. Thus, after a little algebra, the value function (7) reads

$$
\begin{aligned}
V\left(w_{t}, t\right) & =k(1-\theta)\left[w_{0}+\frac{m+c \ln (2)}{2}-w_{0} t-\frac{m+c \ln (2)}{2} t^{2}\right] \\
& =k(1-\theta)\left[w_{t}(1-t)+\frac{m+c \ln (2)}{2}(1-t)^{2}\right] .
\end{aligned}
$$

It is easy to see that (8) is solution of the HJB equation

$$
V_{t}(w, t)+V_{w}(w, t)[m+c \ln (2)]+k(1-\theta) w=0,
$$

with the terminal condition

$$
V(w, 1)=0 .
$$




\section{Models with investment coefficients and loga- rithmic utilities}

In this Section we specify the form of the utility functions of the general model presented in Section 2 as well as the functions $g$ and $h$ in the income dynamic (1). From now on, we assume

$$
\left\{\begin{array}{l}
U\left(y_{1}\right)=a \ln \left(y_{1}\right) \\
C\left(y_{2}, \theta, \alpha\right)=b \frac{\alpha}{\theta} \ln \left(l+y_{2}\right)
\end{array}\right.
$$

and

$$
\left\{\begin{array}{l}
g\left(x_{t}, w_{t}\right)=\left[m+c_{1} \frac{x_{t}}{w_{t}}+c_{2} \frac{\bar{y}_{t}}{w_{t}}\right] w_{t}, \\
h\left(w_{t}\right)=\sigma w_{t}
\end{array}\right.
$$

where

$$
\bar{y}_{t}=(1-p)\left(w_{t}-\theta x_{t}\right)+p\left(w_{t}-\theta x_{t}-f \theta\left(w_{t}-x_{t}\right)\right)
$$

is the effective income (we assume for $p, f, \alpha, \theta$ the same hypotheses of Section 2). Plugging the assumption (9) into (2) the expected well-being becomes

$$
F\left(w_{t}, x_{t}\right)=(1-p) a \ln \left(w_{t}-\theta x_{t}\right)+p a \ln \left(w_{t}-\theta x_{t}-f \theta\left(w_{t}-x_{t}\right)\right)+b \frac{\alpha}{\theta} \ln \left(l+x_{t}\right) .
$$

In this way, we have defined the class (varying the parameters $a>0, b \geq 0, m$, $\left.c_{1} \geq 0, c_{2} \geq 0, \sigma \neq 0, l>0, w_{0}>0\right)$ of stochastic control problems

$$
\begin{gathered}
\sup _{x \in A} E_{0}\left(\int_{0}^{T} F\left(w_{t}, x_{t}\right) d t\right), \\
d w_{t}=\left[m+c_{1} \frac{x_{t}}{w_{t}}+c_{2} \frac{\bar{y}_{t}}{w_{t}}\right] w_{t} d t+\sigma w_{t} d B_{t},
\end{gathered}
$$

with initial endowment $w_{0}>0$.

Notice that the assumptions of Section 2, on $U$ and $C$, are verified. The constant $l$ occurs in the confidence function for technical reasons. It can also be chosen very small, for instance $l=10^{-6}$.

The functions $c_{1} \frac{x_{t}}{w_{t}}$ and $c_{2} \frac{\bar{y}_{t}}{w_{t}}$ in (13) account for the effect respectively of public and private investments on the trend of income. The former is an indirect effect, expressing that the taxpayer is motivated to declare her income by the (expected) positive effect of public investments. The latter effect is linked to the effective income $\bar{y}_{t}$. We may indicate the weights $c_{1}, c_{2}$, respectively, as public and private investment coefficients. The constant $m$ expresses the other factors that drive the expected trend of the income.

Notice that we have chosen the linear form for the investments, but our analysis remains valid also for other functional forms (for instance, for the logarithmic form $c_{1} \ln \left(1+\frac{x_{t}}{w_{t}}\right), c_{2} \ln \left(1+\frac{\bar{y}_{t}}{w_{t}}\right)$, which models decreasing marginal effectiveness of the investments). 


\subsection{Analysis of the (rewritten) models}

In order to study the analytical properties of problem (12), in the following we rewrite the model with investment coefficients by using a transformation of variables. In this way we can bring back the problem to classical theorems of stochastic control. In particular, we demonstrate that the HJB equation has a solution, unique in a space of polynomial growth functions, and this solution, under suitable hypotheses, is equal to the value function.

Let us consider the transformation of variables

$$
\left\{\begin{array}{l}
z_{t}=\frac{x_{t}}{w_{t}}, \\
v_{t}=\ln \left(w_{t}\right) .
\end{array}\right.
$$

The expected well-being (11) becomes

$$
\begin{aligned}
& G\left(v_{t}, z_{t}\right)=F\left(e^{v_{t}}, z_{t} e^{v_{t}}\right) \\
& =(1-p) a \ln \left(e^{v_{t}}-\theta z_{t} e^{v_{t}}\right)+p a \ln \left(e^{v_{t}}-\theta z_{t} e^{v_{t}}-f \theta\left(e^{v_{t}}-z_{t} e^{v_{t}}\right)\right)+b \frac{\alpha}{\theta} \ln \left(l+z_{t} e^{v_{t}}\right) \\
& =(1-p) a\left[v_{t}+\ln \left(1-\theta z_{t}\right)\right]+p a\left[v_{t}+\ln \left(1-\theta z_{t}-f \theta\left(1-z_{t}\right)\right)\right]+b \frac{\alpha}{\theta} \ln \left(l+z_{t} e^{v_{t}}\right) .
\end{aligned}
$$

Applying Itô's formula, we obtain

$$
\begin{aligned}
d v_{t} & =d \ln \left(w_{t}\right)=\frac{1}{w_{t}}\left[m+c_{1} \frac{x_{t}}{w_{t}}+c_{2} \frac{\bar{y}_{t}}{w_{t}}\right] w_{t} d t-\frac{1}{2} \frac{1}{w_{t}^{2}} \sigma^{2} w_{t}^{2} d t+\frac{1}{w_{t}} \sigma w_{t} d B_{t} \\
& =\left[m+c_{1} z_{t}+c_{2} \frac{\bar{y}_{t}}{e^{v_{t}}}-\frac{1}{2} \sigma^{2}\right] d t+\sigma d B_{t},
\end{aligned}
$$

with

$$
\bar{y}_{t}=(1-p)\left(e^{v_{t}}-\theta z_{t} e^{v_{t}}\right)+p\left(e^{v_{t}}-\theta z_{t} e^{v_{t}}-f \theta\left(e^{v_{t}}-z_{t} e^{v_{t}}\right)\right) .
$$

Let us observe that we used Itô's formula without the hypotheses of Itô's lemma (because the logarithmic function is defined only on positive numbers). But if a process $v_{t}$ verifies the stochastic differential law (15), then $e^{v_{t}}$ verifies the law (13) (thus, we can apply Itô's lemma to the exponential function).

By using the transformation of variables (14), therefore, the stochastic control problem (12) and the income dynamic (13) become respectively

$$
\sup _{z \in A^{\prime}} E_{0}\left(\int_{0}^{T} G\left(v_{t}, z_{t}\right) d t\right)
$$

and

$$
d v_{t}=\left[m+c_{1} z_{t}+c_{2} \frac{\bar{y}_{t}}{e^{v_{t}}}-\frac{1}{2} \sigma^{2}\right] d t+\sigma d B_{t}
$$


where $A^{\prime}$ is the set of the admissible controls, defined as

$$
A^{\prime}=\left\{\begin{array}{l}
z(t, \omega):[0, T] \times \Omega \rightarrow \mathbb{R} \text { such that }: \\
\text { 1) } z_{t}(\omega) \text { is measurable with respect to } \mathscr{F}_{t} \text { and } \mathscr{B}(\mathbb{R}), \text { for all } t . \\
\text { 2) There exists a Markov process v solution of the stochastic } \\
\text { differential law, pathwise unique. } \\
\text { 3) } 0 \leq z_{t} \leq 1(\text { in }[0, T] \times \Omega) . \\
\text { 4) } E_{0}\left(\int_{0}^{T} G\left(v_{t}, z_{t}\right) d t\right) \text { exists. } \\
5) z \text { is progressively measurable. }
\end{array}\right\}
$$

(for technical reasons, now we require that the admissible controls are progressively measurable).

The value function of the problem is

$$
R\left(v_{t}, t\right)=\sup _{z \in A^{\prime}} E_{t}\left(\int_{t}^{T} G\left(v_{s}, z_{s}\right) d s\right),
$$

and the related HJB equation is

$$
\begin{aligned}
\sup _{z(\text { with } 0 \leq z \leq 1)} & {\left[G(v, z)+R_{v}(v, t)\left[m+c_{1} z+c_{2} \frac{\bar{y}}{e^{v}}-\frac{1}{2} \sigma^{2}\right]\right.} \\
& \left.+\frac{1}{2} R_{v v}(v, t) \sigma^{2}+R_{t}(v, t)\right]=0
\end{aligned}
$$

with terminal condition

$$
R(v, T)=0 .
$$

The following theorem summarizes some important properties related to the HJB equation and to the solution of the stochastic control problem (19).

\section{Theorem 1.}

1. There exists a solution $\bar{R}(v, t)$ of the HJB equation (20) (with terminal condition (21)), unique in the space of functions $C^{2,1}(\mathbb{R} \times[0, T]) \cap C_{p}(\mathbb{R} \times[0, T])$ $\left(C_{p}(\mathbb{R} \times[0, T])\right.$ is the set of continuous functions $\phi$ on $\mathbb{R} \times[0, T]$, satisfying the polynomial growth condition $|\phi(v, t)| \leq k\left(1+|v|^{h}\right)$ for some constants $\left.k, h\right)$.

2. We have, for all $v, t$,

$$
\bar{R}(v, t) \geq R(v, t)
$$

(namely, the solution of the HJB equation is greater than or equal to the value function). Furthermore, if there exists an admissible control process $\bar{z}_{t}$ that (for almost all $(t, \omega) \in[0, T] \times \Omega)$ maximizes

$$
G\left(\bar{v}_{t}, z\right)+\bar{R}_{v}\left(\bar{v}_{t}, t\right)\left[m+c_{1} z+c_{2} \frac{\bar{y}_{t}}{e^{\bar{v}_{t}}}-\frac{1}{2} \sigma^{2}\right]
$$


(where $\bar{v}_{t}$ is the solution of (18) corresponding to $\bar{z}_{t}$ and with $\bar{y}_{t}$ as in (16)), then, for all $v, t$,

$$
\bar{R}(v, t)=R(v, t)
$$

(namely, the solution of the HJB equation is equal to the value function) and $\bar{z}_{t}$ is an optimal control process.

Proof. In order to demonstrate the first part of the theorem, let us observe that problem (19) verifies the hypotheses of Theorem 4.3 in Fleming and Soner (2006, p. 163) (see also Theorem 6.2 in Fleming and Rishel, 1975, p. 169), which ensures the existence of a solution to the HJB equation, with uniqueness in the space of (polynomial growth) functions $C^{2,1}(\mathbb{R} \times[0, T]) \cap C_{p}(\mathbb{R} \times[0, T])$. In fact, the HJB equation (20) is uniformly parabolic (because $\sigma^{2}>0$ ), $G, G_{v}$ have polynomial growth (with respect to $v$ and $z$ ), and the control processes have values in a compact set ( $[0,1])$.

The second part follows from a verification theorem (see Theorem 3.1 in Fleming and Soner, 2006, p. 157).

Theorem 1 reduces the analysis of the optimal stochastic control problem (19) to solve the HJB equation (20) and to find the process $\bar{z}_{t}$ that maximizes (22). We perform these task in the next Section. This will allow us to study the (stochastic) total income $\bar{X}_{\text {Tot }}$ (see (3)) that the taxpayer expects to declare, given by

$$
\bar{X}_{T o t}=\int_{0}^{T} \bar{z}_{t} e^{\bar{v}_{t}} d t
$$

obtained inverting the transformation of variables (14), with $\bar{v}_{t}$ solution of (18) corresponding to $\bar{z}_{t}$.

\subsection{Computational example}

Here we aim at showing how the theoretical results presented in the previous Section can be used to analyze the taxpayer compliance behavior. More precisely, we numerically solve the stochastic control problem (17) and we perform a Monte Carlo simulation in order to provide an estimate of the mean and the variance of $\bar{X}_{\text {Tot }}$.

In order to do that we consider for example the following values of the parameters of the (rewritten) model (for simplicity, in the case with null confidence)

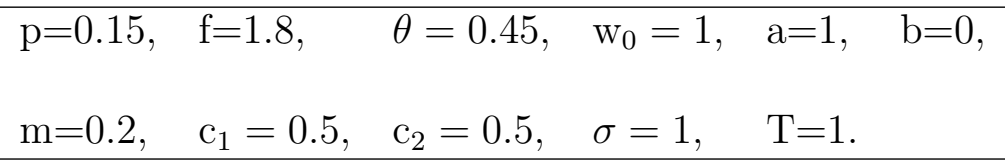


Through a suitable algorithm, the maximization problem in the HJB equation (20) is solved and the value function (as a solution of the corresponding partial differential equation, numerically solved) is estimated (see figure 1 ). Then $10 \times n$ random variables with standard normal distribution are simulated and $n$ (discretized) paths of $v_{t}$ (each consisting of 10 values in equally spaced times, from $t=0.1$ to $t=1$ ) are determined, together with the corresponding $n$ paths of $x_{t}$. For each path of $x_{t}$ the associated value of $\bar{X}_{T o t}$ is computed through a numerical integration. Finally, by means of a Monte Carlo method, the estimators $M=\frac{\sum_{j=1}^{n} \bar{X}_{T o t_{j}}}{n}$ and $S^{2}=\frac{\left(\sum_{j=1}^{n} \bar{X}_{T_{0 t}}^{2}\right)-n M^{2}}{n-1}$ respectively for the mean and the variance of $\bar{X}_{\text {Tot }}$ are calculated (see also examples in (Spartà, 2016)), together with an approximate confidence interval (at the level of $95 \%) I=\left[M-1.96 \sqrt{\frac{S^{2}}{n}}, M+1.96 \sqrt{\frac{S^{2}}{n}}\right]$ (with the same procedure, higher-order moments of $\bar{X}_{\text {Tot }}$ and the histogram of its distribution could be estimated).

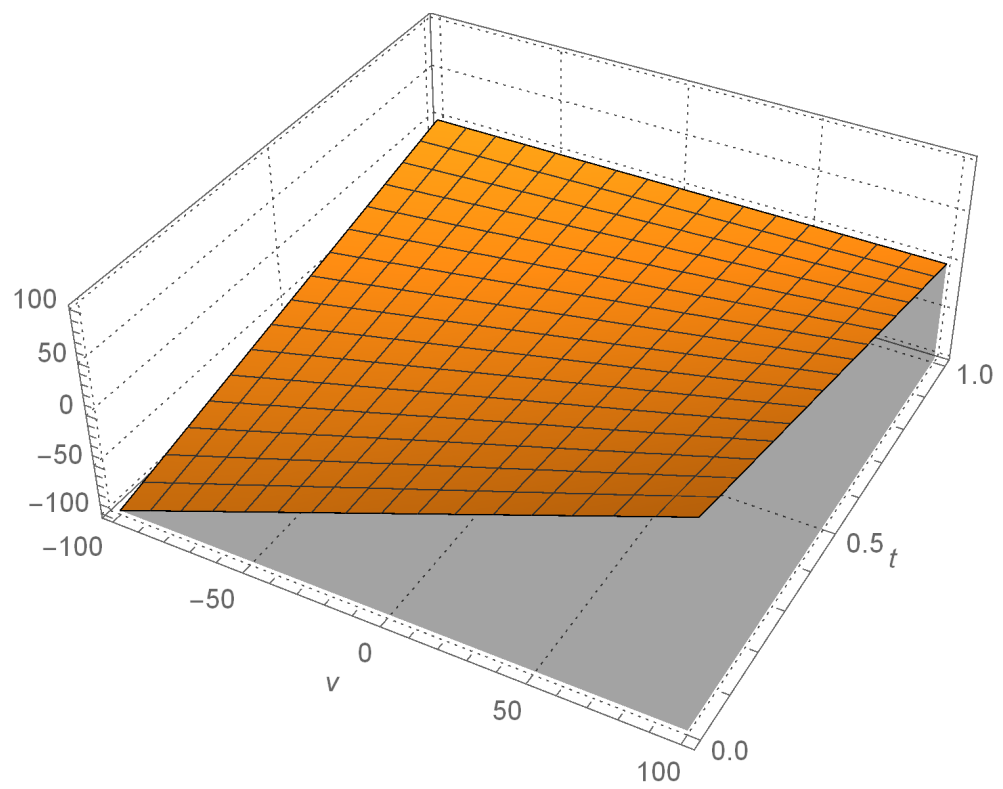

Figure 1: The value function $R(v, t)$.

Executing the algorithm with $n=10^{4}$ simulations of paths, it was obtained

$$
M=0.182709, S^{2}=0.00541982, I=[0.181266,0.184152] .
$$

Varying the parameters of the problem, the following tables were obtained.

Table 1 shows how the estimated mean $M$ reacts to changes in the magnitude of the public investment coefficient $c_{1}$, for different values of the utility parameter $a$ (see (9) and (10)). We can observe that, increasing the coefficient $c_{1}$, the (expected) total declared income tends to increase as well (in both cases $a=1$ and $a=1.5$ ). Therefore, if the effect of public investment on taxpayer income raises, then she is more motivated to tax compliance. 


\begin{tabular}{|c|c|c|c|c|c|}
\hline $\mathrm{c}_{1}$ & 0.25 & 0.5 & 0.75 & 1 & 1.25 \\
\hline 1 & 0.000 & 0.183 & 0.553 & 0.954 & 1.325 \\
\hline 1.5 & 0.000 & 0.183 & 0.560 & 0.947 & 1.303 \\
\hline
\end{tabular}

Table 1: Sensitivity of $M$ with respect to $a$ and $c_{1}$.

\begin{tabular}{|l|l|l|l|}
\hline \multicolumn{1}{|r|}{$\mathrm{f}$} & 1.6 & 1.7 & 1.8 \\
$\mathrm{p}$ & & & \\
\hline 0.14 & 0.030 & 0.072 & 0.146 \\
\hline 0.15 & 0.045 & 0.097 & 0.183 \\
\hline 0.16 & 0.063 & 0.125 & 0.224 \\
\hline
\end{tabular}

Table 2: Sensitivity of $M$ with respect to $p$ and $f$.

In Tables 2 and 3 the values of the estimated mean $M$ are reported, varying the probability $p$ of being discovered or the fine $f$, or the coefficient of private investment $c_{2}$. Notice that an increase in the values of the enforcement parameters $p$ or $f$ leads to an increase in the (expected) total declared income. On the other hand, $M$ tends to decrease as the coefficient $c_{2}$ increases. Intuitively, the higher is $c_{2}$, the greater is the impact of the private investments on taxpayer's income (cfr. (13)). Thus, she reduces tax compliance to finance private investments.

Table 4 displays the values of the estimated mean $M$ when $\sigma$ and $\theta$ are varied. Notice that as the tax rate $\theta$ increases, the (expected) total declared income tends to decrease, i.e. there is a positive relationship between evasion and the tax rate. Furthermore, varying $\sigma$ (cfr. (13)), the values of $M$ seem to stay rather stable.

Finally, Table 5 shows how the values of the estimated variance $S^{2}$ react to changes in the values of $\sigma$, for different values of initial endowment $w_{0}$. Notice that if the constant $\sigma$ increases, then the variance of the total declared income tends to increase as well (in both cases $w_{0}=1$ and $w_{0}=e^{\frac{1}{2}}$ ). These results suggest that changes in the income uncertainty, while have little impact on the mean of $M$, make more volatile the total declared income.

\begin{tabular}{|c|c|c|c|}
\hline $\mathrm{c}_{2}$ & 0.25 & 0.5 & 0.75 \\
\hline 0.14 & 0.237 & 0.146 & 0.064 \\
\hline 0.15 & 0.274 & 0.183 & 0.097 \\
\hline 0.16 & 0.315 & 0.224 & 0.136 \\
\hline
\end{tabular}

Table 3: Sensitivity of $M$ with respect to $p$ and $c_{2}$. 


\begin{tabular}{|l|l|l|l|}
\hline \multicolumn{1}{|r|}{$\theta$} & 0.35 & 0.4 & 0.45 \\
\hline 1 & 0.290 & 0.189 & 0.183 \\
\hline 1.5 & 0.289 & 0.190 & 0.184 \\
\hline 2 & 0.290 & 0.191 & 0.183 \\
\hline
\end{tabular}

Table 4: Sensitivity of $M$ with respect to $\sigma$ and $\theta$.

\begin{tabular}{|c|c|c|c|c|c|}
\hline $\begin{array}{ll} & \sigma \\
\mathrm{w}_{0} & \end{array}$ & 1 & 1.25 & 1.5 & 1.75 & 2 \\
\hline 1 & 0.005 & 0.009 & 0.016 & 0.023 & 0.033 \\
\hline $\mathrm{e}^{\frac{1}{2}}$ & 0.015 & 0.027 & 0.046 & 0.060 & 0.094 \\
\hline
\end{tabular}

Table 5: Sensitivity of $S^{2}$ with respect to $w_{0}$ and $\sigma$.

\section{Conclusions}

In this paper we propose a general stochastic control model to analyze the compliance behaviour of a taxpayer endowed with a stochastic income. We assume that the income dynamic is driven by economic factors linked to declared income (like public and private investments). As a particular case (weakening the hypotheses on $U$ ), we consider a model that allows to solve a paradox in the taxpayer's choice with risk neutrality. Then we consider a class of models with public and private investment coefficients, that we study in their analytical properties with a suitable transformation of variables, offering also a computational example.

With regard to the methodological perspective, in this paper we aim to provide a framework that allows to examine the taxpayer's compliance. Therefore, in this context we do not analyse economic data.

In a possible development, the parameters can be calibrated on real data, also in order to check the effectiveness of the model results.

Another improvement would be the deepening of the mathematical properties of the models (for instance, in the case of models with investment coefficients, the possibility of using constant relative risk aversion utilities, different from the logarithmic function). Finally, it would be nice to use these models as part of general equilibrium models.

\section{References}

[1] M. G. Allingham, A. Sandmo. Income tax evasion: A theoretical analysis. Journal of Public Economics 1, 323-338. 1972.

[2] J. Andreoni, B. Erard, J.S. Fenstein. Tax compliance. Journal of Economic Literature 36, 818-860. 1998. 
[3] M. Bordignon. A fairness approach to income tax evasion. Journal of Public Economics 52, 345-362. 1993.

[4] F. Célimène, G. Dufrénot, G. Mophou, G. N'Guérékata. Tax evasion, tax corruption and stochastic growth. Economic Modelling 52, 251-258. 2016.

[5] R. Cerqueti, R. Coppier. A game theoretical analysis of the impact of income inequality and ethnic diversity on fiscal corruption. Annals of Operations Research. 2014.

[6] R. Cerqueti, R. Coppier. Economic growth, corruption and tax evasion. Economic Modelling 28, 489-500. 2011.

[7] B.-L. Chen. Tax evasion in a model of endogenous growth. Review of Economic Dynamics 6, 381-403. 2003.

[8] F. A. Cowell. Cheating the Government. The Economics of Evasion. The MIT Press, Cambridge, MA. 1990.

[9] R. Dzhumashev. Corruption, uncertainty and growth. Discussion Paper 15/07, Department of Economics, Monash University. 2007.

[10] R. Dzhumashev, E. Gahramanov. Can we tax the desire for tax evasion? Discussion Paper 28/08, Department of Economics, Monash University. 2008.

[11] R. Dzhumashev, E. Gahramanov. Comment on 'A dynamic portfolio choice model of tax evasion: Comparative statics of tax rates and its implication for economic growth'. Journal of Economic Dynamics and Control 35, 253-256. 2011.

[12] B. Erard, J.S. Fenstein. The role of moral sentiments and audit perceptions in tax compliance. Public Finance 49, 70-89. 1994.

[13] W. H. Fleming, R. W. Rishel. Deterministic and stochastic optimal control. Springer-Verlag, 1975.

[14] W. H. Fleming, H. M. Soner. Controlled Markov processes and viscosity solutions. Second edition. Springer, 2006.

[15] J. P. F. Gordon. Individual morality and reputation costs as deterrents to tax evasion. European Economic Review 33, 797-805. 1989.

[16] R. Levaggi, F. Menoncin. Optimal dynamic tax evasion. Journal of Economic Dynamics and Control 37, 2157-2167. 2013.

[17] W.-Z. Lin, C. C. Yang. A dynamic portfolio choice model of tax evasion: Comparative statics of tax rates and its implication for economic growth. Journal of Economic Dynamics and Control 25, 1827-1840. 2001. 
[18] M. O'Doherty. Thinking and Learning in the Tax Evasion Game. Fiscal Studies, Vol. 35, No. 3, pp. 297-339. 2014.

[19] A. Sandmo. The theory of tax evasion: A retrospective view. National Tax Journal, Vol. 58, No. 4, pp. 643-663. 2005.

[20] G. T. Spartà. Tax evasion and confidence in institutions: A theoretical model. 2015. Available at SSRN.

[21] G. T. Spartà. Tax evasion and confidence in institutions: Two expectations models. 2016. To appear on International Journal of Computational Economics and Econometrics.

[22] B. Torgler. To evade taxes or not to evade: that is the question. Journal of Socio-Economics 32, 283-302. 2003.

[23] S. Yitzhaki. A note on Income tax evasion: A theoretical analysis. Journal of Public Economics 3, 201-202. 1974. 